\title{
Low-frequency direct cortical stimulation of left superior frontal gyrus enhances working memory performance
}

Sankaraleengam Alagapan ${ }^{1,7}$, Caroline Lustenberger ${ }^{1}$, Eldad Hadar $^{2}$, Hae Won Shin ${ }^{2,3}$, and Flavio Fröhlich ${ }^{1,3,4,5,6,7}$

Correspondence should be addressed to: Flavio Fröhlich, 115 Mason Farm Rd. NRB 4109F, Chapel Hill, NC. 27599. Email: flavio_frohlich@med.unc.edu

1 Department of Psychiatry, University of North Carolina at Chapel Hill, Chapel Hill NC 27599, USA

2 Department of Neurosurgery, University of North Carolina at Chapel Hill, Chapel Hill NC 27599, USA

3 Department of Neurology, University of North Carolina at Chapel Hill, Chapel Hill NC 27599, USA

4 Department of Biomedical Engineering, University of North Carolina at Chapel Hill, Chapel Hill NC 27599, USA

5 Department of Cell Biology and Physiology, University of North Carolina at Chapel Hill, Chapel Hill NC 27599, USA

6 Neuroscience Center, University of North Carolina at Chapel Hill, Chapel Hill NC 27599, USA 7 Carolina Center for Neurostimulation, University of North Carolina at Chapel Hill, Chapel Hill NC 27599, USA

Authorship Statement: SA, HS, and FF designed the experiments; SA, EH, and HS performed the electrophysiological recordings; SA, CL analyzed the data; and SA, CL, EH, HS, and FF prepared the manuscript. 


\section{Abstract}

The neural substrates of working memory are spread across prefrontal, parietal and cingulate cortices and are thought to be coordinated through low frequency cortical oscillations in the theta $(3-8 \mathrm{~Hz})$ and alpha $(8-12 \mathrm{~Hz})$ frequency bands. While the functional role of many subregions have been elucidated using neuroimaging studies, the role of superior frontal gyrus (SFG) is not yet clear. Here, we combined electrocorticography and direct cortical stimulation in three patients implanted with subdural electrodes to assess if superior frontal gyrus is indeed involved in working memory. We found left SFG exhibited task-related modulation of oscillations in the theta and alpha frequency bands specifically during the encoding epoch. Stimulation at the frequency matched to the endogenous oscillations resulted in reduced reaction times in all three participants. Our results support the causal role of SFG in working memory and suggest that SFG may coordinate working memory through low-frequency oscillations thus bolstering the feasibility of targeting oscillations for restoring cognitive function. 


\section{Introduction}

2 Working memory (WM), the ability to flexibly maintain and manipulate information for a short

3 period of time, forms an important component of cognition. It supports other higher-order cognitive

4 functions and has been tightly linked to fluid intelligence [1, 2]. Impairment in WM accompanies

5 many neurological and psychiatric disorders and significantly reduces the quality of life of affected

6 patients [3-7]. A mechanistic understanding of the causal role of circuit dynamics in WM will open

7 new therapeutic avenues.

8 Functional imaging studies have revealed that the neural substrate of WM is spread across

9 multiple cortical regions including dorsolateral prefrontal cortex, posterior parietal cortex and

10 anterior cingulate cortex. While early studies have suggested superior frontal gyrus (SFG) to be

11 involved in working memory [8-10], subsequent studies have often found the middle frontal gyrus

12 (MFG) to be the key node in working memory [11-15]. However, lesions in SFG have been shown

13 to result in working memory deficits [16]. In addition, electroencephalography (EEG) and

14 magnetoencephalography (MEG) studies have shown that oscillations in the theta frequency

15 band $(4-8 \mathrm{~Hz})$ observed on fronto-central regions [17-21] coordinate working memory. The

16 source of these oscillations is thought to be medial prefrontal cortex which includes SFG.

17 Modulations in WM performance by non-invasive brain stimulation like repetitive transcranial

18 magnetic stimulation (rTMS) [22, 23] and transcranial alternating current stimulation (tACS) [24-

19 27] targeting prefrontal cortex also provide indirect evidence for the role of SFG in WM

20 performance.

21 Electrocorticography (ECoG) allows identification of activity signatures at temporal scale of a few

22 milliseconds with a spatial resolution of a few centimeters is an ideal tool to map functions of

23 cortical regions. Direct cortical stimulation, in which stimulation is applied through ECoG

24 electrodes, allows for focal probing of cortex providing additional information through reversible

25 microlesions [28]. Combined recording and stimulation with implanted electrodes have greatly

26 contributed to revealing the substrate of long-term memory [29-32]. Low amplitude periodic

27 stimulation at $10 \mathrm{~Hz}$ has been demonstrated to engage ongoing cortical oscillations in a state-

28 dependent manner and enhance oscillation strength measured by signal power [33]. In this study, 
we employed a similar experimental paradigm to delineate the role of SFG on working memory.

30 We present results from three participants with subdural electrodes over left and right SFG in whom we assessed the electrophysiological signatures of SFG and applied periodic stimulation during a verbal working memory task. We found that left SFG exhibited a task-related modulation in oscillation power and stimulation matched to the frequency of oscillation resulted in an improvement in working memory performance.

\section{Results}

We leveraged the access to ECoG signals in three patients with epilepsy undergoing long term monitoring in the Epilepsy monitoring unit at the N.C. Neurosciences Hospital, UNC Medical Center, Chapel Hill. The participants (P1, P2 and P3) had electrodes over frontal, temporal and parietal regions on both hemispheres (Figure 1A). The participants performed a Sternberg verbal working memory task that has been previously used in ECoG research [34, 35] (Figure 1B). The cognitive load, measured by the number of items (English letters) in a list to be held in memory (3, 4, or 5 for $\mathrm{P} 1$ and 3 or 5 for $\mathrm{P} 2$ and 5 or 7 for $\mathrm{P} 3$ ), was varied randomly for each trial. In participant

$\mathrm{P} 1$, we observed an increase in reaction times with increasing cognitive load (list length 3: $824 \pm$ $31 \mathrm{~ms}$, list length 4: $1119 \pm 105 \mathrm{~ms}$, list length 5: $1140 \pm 78 \mathrm{~ms}$ ) in the sham trials (Linear model factor list length: $F(2,34)=4.864 ; p=0.014)$. In participant $P 2$, who performed a separate baseline session of the task without stimulation, there was no significant difference between reaction times for different cognitive loads $(F(1,20)=0.060 ; p=0.809)$. In participant $P 3$, who also performed a separate baseline session without stimulation, there was a significant effect of cognitive load (Linear mixed model factor list length: $F(1,45)=4.646 ; p=0.036)$. The reaction time for trials with

5 items in the list was lower than trials with 7 items in the list (785 $\pm 26 \mathrm{~ms}$ vs $902 \pm 48 \mathrm{~ms}$ ). 

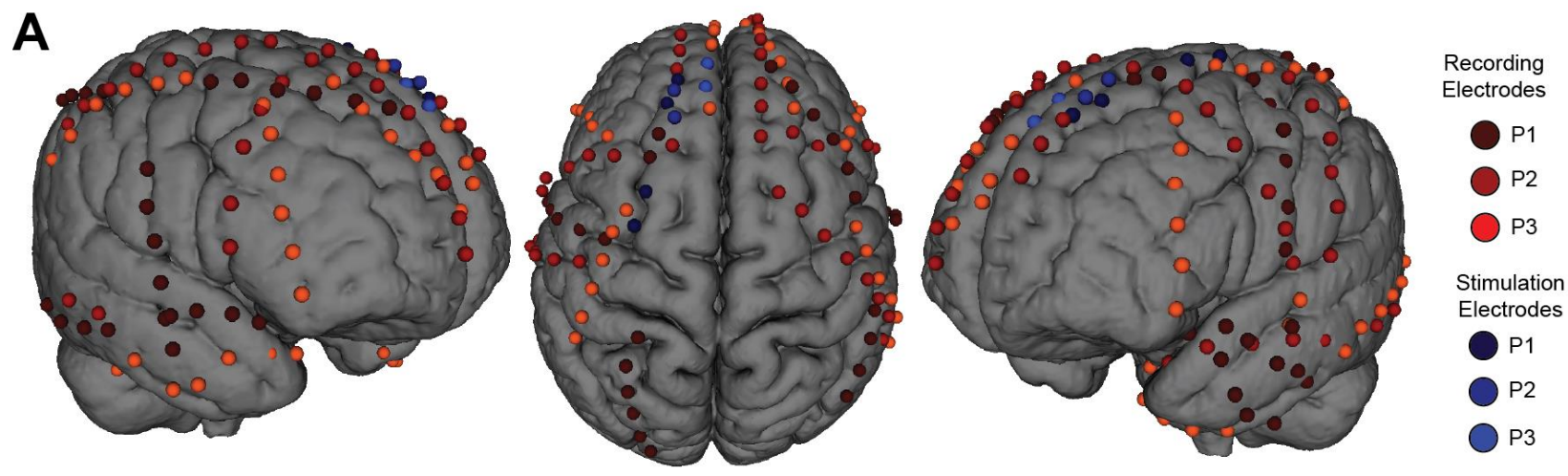

B

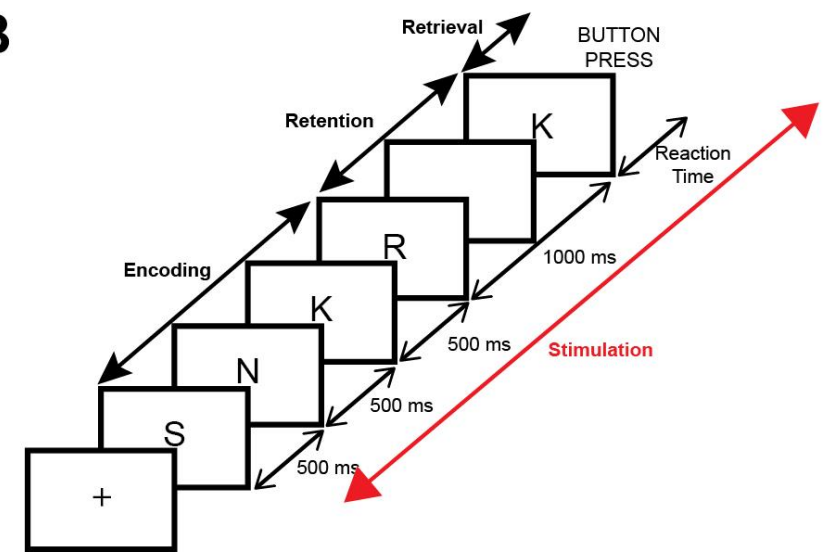

C

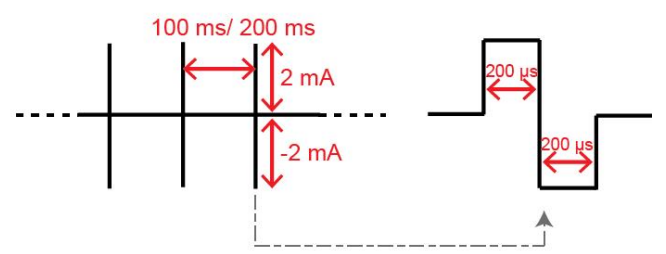

Figure 1. (A) Surface model showing the coverage of electrodes for the three participants. (B) Schematic of a single trial of the working memory task used. The task consisted of 3 epochs - Encoding, Retention and Retrieval. Stimulation was applied through the entire trial. (C) Schematic of the periodic pulse stimulation. Stimulation consisted of train of biphasic pulses $400 \mu \mathrm{s}$ in duration every $100 \mathrm{~ms}$ (P1 and P2) or $200 \mathrm{~ms}$ (P3) for $5 \mathrm{~s}$.

53 Oscillations in the theta $(3-8 \mathrm{~Hz})$ and alpha $(8-12 \mathrm{~Hz})$ bands have been shown to be modulated

54 during working memory tasks [19, 34, 36]. Spectral analysis revealed oscillations with a peak frequency around $5 \mathrm{~Hz}$ in P1 and P3 and $9.5 \mathrm{~Hz}$ in P2. To assess if these observed oscillations were modulated by the task, we computed power spectra for baseline, encoding and retention epochs when no stimulation was being delivered (sham trials in P1 and baseline session trials in P2 and P3). Modulation indices were computed relative to baseline epoch. The retrieval epoch was not included in analysis as the epoch may be confounded with action planning and action. We

60 found that electrodes in frontal, temporal and parietal regions exhibited an enhancement of power

61 relative to baseline during sham trials in the theta band $(3-8 \mathrm{~Hz})$ in $\mathrm{P} 1$ and $\mathrm{P} 3$ and in alpha band $(8-10 \mathrm{~Hz})$ in P2 (one sample t-test with FDR correction; $\mathrm{p}<0.05)$. Specifically, electrodes over the left superior frontal gyrus (ISFG) exhibited the task relevant enhancement of oscillation across 
bioRxiv preprint doi: https://doi.org/10.1101/302588; this version posted April 17, 2018. The copyright holder for this preprint (which was not certified by peer review) is the author/funder, who has granted bioRxiv a license to display the preprint in perpetuity. It is made available under aCC-BY-NC-ND 4.0 International license.

A
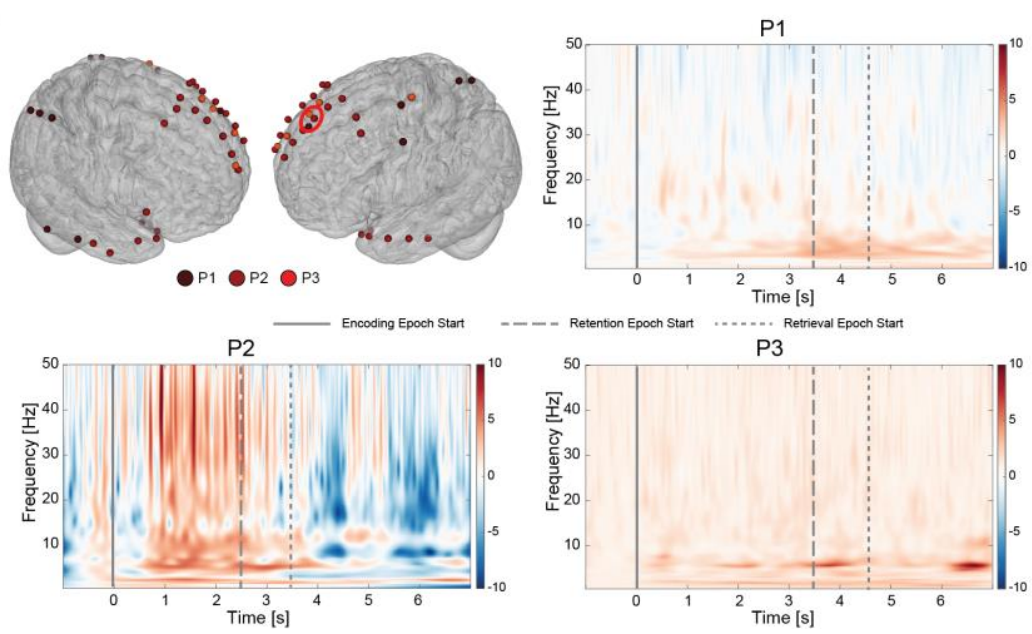

B

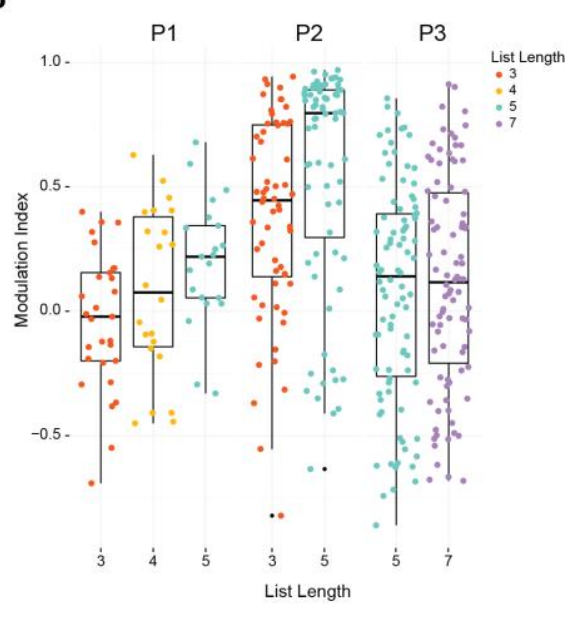

Figure 2. (A) Cortical model showing electrodes that exhibited task-related modulation. Red circle denotes the three electrodes in ISFG whose event related spectral perturbation are plotted. observed in left superior frontal gyrus electrodes during sham trials for P1 and baseline session trials for P2 and P3 indicating the modulation of signal in the band $3-12 \mathrm{~Hz}$. Hot (red) colors indicate an increase and cold (blue) colors indicate a decrease in signal power relative to baseline. (B) Modulation indices during encoding epoch across all ISFG electrodes that exhibited significant task related modulation of signal power. In P1 and P2 there was a significant difference between modulation indices for list length 3 and list length 5 .

74 To test if targeting oscillations that exhibited task-related modulation in SFG, periodic pulse stimulation was applied between pairs of electrodes over the left SFG. Stimulation consisted of pulse trains $2 \mathrm{~mA}$ in amplitude and 5 seconds in duration (Figure 1C) and the electrode pair being stimulated was randomly changed for each trial. Stimulation and sham trials were randomly interleaved and trial initiation was time-locked to stimulation initiation. Stimulation frequency was $10 \mathrm{~Hz}$ for P1 (chosen a priori), $9 \mathrm{~Hz}$ for $\mathrm{P} 2$ (due to technical issues) and $5 \mathrm{~Hz}$ for P3. A total of two 
different pairs of electrodes in left SFG were stimulated in P1, one pair of electrodes in P2 and P3 (blue electrodes in Figure 1A). During the stimulation session, participants P1, P2 and P3 performed the task in which trials consisted of 3, 4 or 5 items, 3 or 5 items, 5 items respectively. As a first step, the effect of stimulation on reaction times of participants P1 and P2 was analyzed using a linear mixed model with fixed factors list length and stimulation condition and participant as random factor as these participants had trials with 3 and 5 items. While there was no significant effect of stimulation $(F(2,108)=1.042 ; p=0.356)$, there was a significant effect of list length ( $F(1,108)=9.072 ; p=0.003)$ and interaction between list length and stimulation condition ( $F(2,108)=6.536 ; p=0.002)$. Next analysis was restricted to only trials with 5 items and the reaction times of all 3 participants in sham trials were compared with that in stimulation trials. The effect of stimulation was statistically significant (Linear Mixed Model $F(1,97)=13.414 ; p<0.001$ ) with all participants showing a significant decrease in reaction times (P1: $1140 \pm 78$ ms vs $852 \pm$ $111 \mathrm{~ms}$; P2: $1188 \pm 93$ ms vs $954 \pm 54$ ms; P3: $841 \pm 48$ ms vs $727 \pm 27 m s$; Figure $3 A$ ) confirmed by post-hoc analysis (Pairwise t-test, $\mathrm{p}<0.05$ ). Analysis of accuracy using chi-squared tests did not reveal any significant interactions (Figure 3B) suggesting stimulation served to reduce reaction times without affecting accuracy.

In most studies involving electrical stimulation, artifacts caused by stimulation prevent the analysis of electrophysiological signals during stimulation. To overcome this, we developed an independent component analysis (ICA) based method (see Methods and Experimental Procedures). Stimulation artifacts were sufficiently suppressed (Figure S1) allowing us to study the signals in the frequency band of interest. Power spectra and modulation indices in the endogenous oscillation frequency band $(3-8 \mathrm{~Hz}$ in $\mathrm{P} 1$ and $\mathrm{P} 3$ and $8-12 \mathrm{~Hz}$ in $\mathrm{P} 2)$ were computed as described before. Analysis of modulation indices of the electrodes over ISFG (restricted to trials with 5 items in the list) across all participants did not reveal any significant effect of stimulation 104 (Figure 3C; Linear mixed model factor condition $F(1,459)=0.612 ; p=0.434$ ). To explore the effects of stimulation on other regions that exhibited modulation of task-relevant oscillations, we ran analysis on individual participant data including list length as a factor. In P1, stimulation induced a differential change in modulation indices (Linear mixed model factor condition $F(1,672)$ 
$108=20.827 ; p<0.001$, factor list length $F(1,672)=15.793 ; p=0.001$, interaction $F(1,672)=10.536$;

$109 \mathrm{p}=0.004)$. Further analysis revealed that there was a significant effect of stimulation in trials with

1105 items in list, with stimulation inducing a decrease in modulation indices (Linear mixed model

111 factor condition $\mathrm{F}(1,305=27.742 ; \mathrm{p}<0.001)$. Similarly in $\mathrm{P} 2$, stimulation induced a difference

112 change in modulation indices (Linear mixed model factor condition $F(1,1738)=0.495 ; p=0.482$,

113 factor list length $F(1,1738)=33.190 ; p<0.001$, interaction $F(1,1738)=11.134 ; p<0.001)$.

114 Stimulation caused significant decrease in modulation indices in trials with 3 items (Factor

115 condition $F(1,908)=9.04 ; p=0.003$ ) while stimulation caused a trend-level significant increase in 116 modulation indices in trials with 5 items (Factor condition $F(1,830)=3.13 ; p=0.077$ ). There was

117 no significant effect of stimulation in P3 (Factor condition $F(1,215)=0.005 ; p=0.946$ ).

A

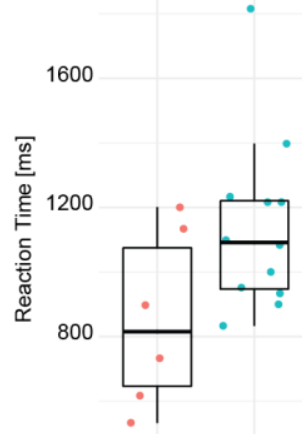

P1

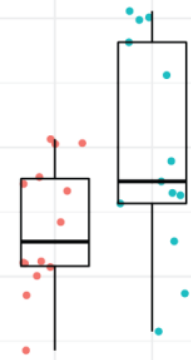

P2
B

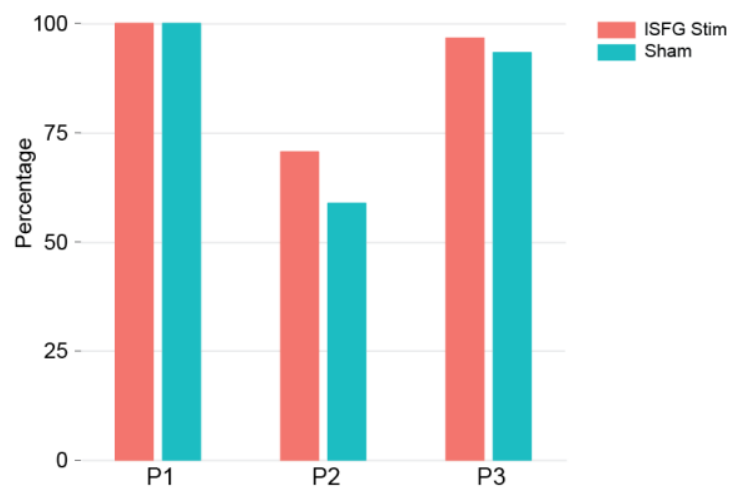

C

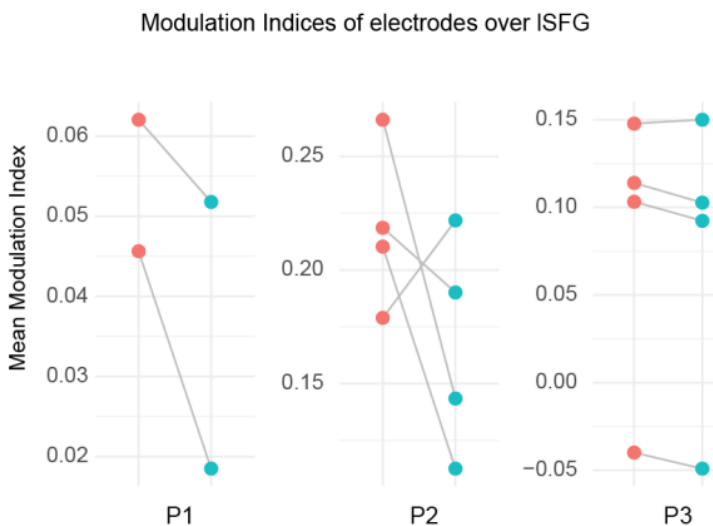

118

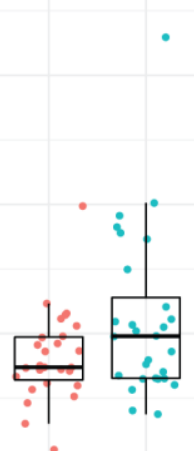

P3

\section{D}

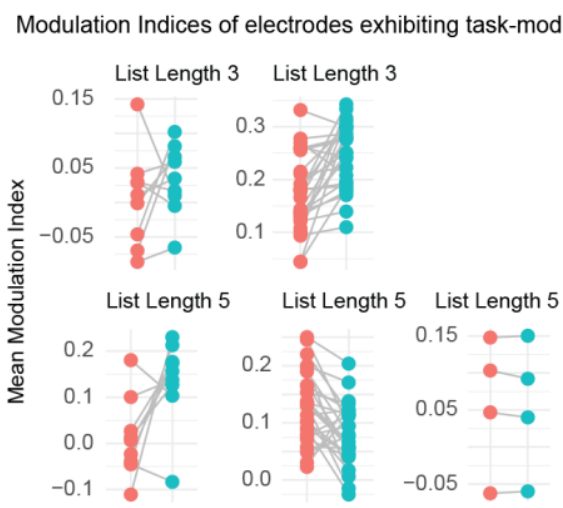

P1

P2

P3 
Figure 3. (A) Reaction times in trials with 5 items showing a decrease with stimulation. (B) Accuracy was not affected by stimulation (C) Stimulation did not result in any changes in modulation indices in electrodes over ISFG. (D) Differential effect of stimulation on modulation indices in electrodes that exhibited taskrelevant modulation of low frequency oscillations.

\section{Discussion}

121 In this study, we show evidence for the role of superior frontal gyrus (SFG) in working memory using a combination of ECoG and DCS. Electrodes over left SFG exhibited modulation of cortical oscillations in the canonical theta and alpha frequency bands. The degree of modulation, measured using modulation index, depended on the cognitive load, specifically in the encoding epoch. Stimulation of ISFG with frequency matched to the fundamental frequency or harmonic of the endogenous oscillations, led to an enhancement in working memory performance. However, analysis of data obtained during stimulation did not provide any conclusive evidence for modulation of task-relevant oscillations. Taken together, the results suggest SFG may be an important node in brain network that coordinates working memory.

While there is an abundance of evidence for the role of middle frontal gyrus (MFG; Brodmann Area 9/46) in working memory from neuroimaging studies [11, 15, 37, 38], the role of SFG is not clear. There have been a few neuroimaging studies that suggest SFG may be involved in working memory [8-10, 39]. SFG gray matter volume has been linked to working memory activation in intra-parietal sulcus [40]. The strongest evidence for the role of SFG in working memory has come from a lesion study [16] in which patients with lesions in ISFG exhibited deficits in working memory involving verbal, spatial and face stimuli. Our results strengthen the evidence for SFG's role in working memory. However, the proximal location our stimulation targets to MFG may confound our interpretation of the results. Diffusion tensor tractography has revealed that SFG can be divided into subregions with strong connectivity to ACC, a key node in cognitive control network and MFG, a key node in executive control network [41]. As both networks are essential to working memory processes [42-44], stimulation of SFG may have distributed effects across multiple regions including MFG. The lack of sufficient coverage of these areas in these three patients 
bioRxiv preprint doi: https://doi org/10.1101/302588; this version posted April 17, 2018. The copyright holder for this preprint (which was not certified by peer review) is the author/funder, who has granted bioRxiv a license to display the preprint in perpetuity. It is made available under aCC-BY-NC-ND 4.0 International license.

143 limited our ability to examine this idea. Previous studies have observed oscillations in the range 3

$144-15 \mathrm{~Hz}$ to be modulated during working memory tasks $[34,45,46]$ and the strength of oscillations 145 to reflect working memory load $[19,35,36]$. Frontal midline theta (FMT) is a commonly observed 146 oscillatory signature in EEG studies of working memory [18] typically in Fz and neighboring 147 electrodes in the 10-20 electrode system. The sources of FMT are thought to include lateral PFC 148 and ACC [47]. The theta oscillations we observed in our study may be related to FMT although we 149 did not have any scalp electrodes to confirm this. We found task-related modulation specifically in 150 the encoding period. Analysis of oscillation strength in the retention epoch did not reveal any 151 significant difference between the cognitive loads. This suggests that SFG may play a role that is 152 different from that of MFG/IFG which is known to predominantly be active during the retention 153 epoch [15].

154 To the best of our knowledge, this is the first study where effects of intracranial stimulation on working memory and on oscillation strength were investigated. Periodic pulse stimulation of entorhinal region has been shown to improve performance in a spatial learning task [31].

157 Concurrently there was an increase in theta-phase resetting. In another study, stimulation with very weak sinusoidal currents $(0.01 \mathrm{~mA})$ produced trend level effects in memory performance although no improvement compared to sham was seen [48]. Impairment of performance has been more commonly reported than improvement especially for hippocampal stimulation. One study showed that single pulse stimulation of hippocampus impaired episodic memory [49]. In another study, stimulation at $50 \mathrm{~Hz}$ impaired recognition of specific stimuli depending on whether left or right hippocampus was stimulated [50]. More recently, stimulation of entorhinal/ hippocampal and medial temporal regions was shown to affect both verbal and spatial memory $[32,51]$. One key difference between the studies described above and our current study is the frequency of stimulation used. Often, $50 \mathrm{~Hz}$ was chosen as the stimulation frequency as opposed to the low frequency used in our study. A study that utilized low frequency stimulation showed that stimulation at $5 \mathrm{~Hz}$ resulted in improvement of delayed recall [52]. Another study in which theta burst stimulation ( $100 \mathrm{~ms}$ trains of $0.1 \mathrm{~ms}$ pulses at $200 \mathrm{~Hz}$ repeated 5 times per second) of fornix resulted in improvement of visual-spatial memory [53]. These results suggest that frequency of 
171 stimulation might be crucial to the effects observed. Intracranial stimulation studies have often

172 focused on episodic memory and stimulation of hippocampus. In contrast, non-invasive stimulation

173 studies have focused on working memory specifically and target cortical regions such as dIPFC,

174 PPC, inferior frontal gyrus. Transcranial magnetic stimulation, which produces local

175 suprathreshold effects, i.e., evoking action potentials like those expected in intracranial

176 stimulation, has been shown to enhance working memory performance based on the stimulation

177 frequency, location and specific epoch within the task or before the task [54-61]. It must also be

178 noted that many studies report impairments of working memory and episodic memory by TMS as

179 well [62-65]. Transcranial alternating current stimulation, which likely produces more global

180 subthreshold effects, has been shown to increase performance by targeting dIPFC and PPC [24,

181 26]. The neurophysiological underpinnings of the effects in these studies are often unclear [27,

182 60]. Recently, rTMS applied at theta frequency to left intraparietal sulcus was shown to entrain

183 theta oscillations with a concurrent improvement in auditory working memory [66].

184 As any scientific study, our study has a set of limitations. First, the results presented here are from 185 three participants. The major obstacle in our case was the heterogeneity in electrode distribution as the electrode locations were dictated by clinical needs. Second, although the stimulation frequency was $10 \mathrm{~Hz}$, oscillations in the frequency band $3-8 \mathrm{~Hz}$ were significantly modulated concurrently with changes in WM performance. This discrepancy is hard to reconcile if entrainment is thought to be the underlying mechanism of interaction between stimulation and oscillation $[67,68]$. However, the interaction between stimulation and an ongoing oscillation has 191 been found to be nonlinear and the effects depend on the strength of the prevailing oscillations 192 [33]. When there is a strong ongoing oscillation, stimulation tends to increase the strength of the endogenous oscillation and only in cases where the strength of the oscillation is low, entrainment is possible. This state-dependent effect of stimulation is likely the underlying mechanism in the current study as well. Alternatively, $10 \mathrm{~Hz}$ stimulation may have engaged with the strong $5 \mathrm{~Hz}$ oscillation through subharmonic entrainment as predicted in computational models [69].Third, the present experimental paradigm is limited to applying stimulation during the entire trial due to technical limitations of the FDA-approved cortical stimulator used in the study. This limitation 
precluded us from identifying if stimulation during an epoch within a trial, i.e. encoding or retention, is more effective than stimulation during the entire trial. Moreover, the frequency of stimulation was restricted to a few discrete frequencies that did not allow matching of the stimulation frequency to frequency of endogenous oscillations in P1. Fourth, a limitation of the current study design is that it used only a single stimulation amplitude and stimulation frequency. Given the large parameter space, it is prohibitively difficult to try all possible parameters in studies with limited participant pools as the current study. For P1, we chose stimulation regions based on previous literature due to technical limitations. A more effective strategy was followed for P2 and P3 where we identified electrodes that exhibited task-related modulation in low frequency bands and applied stimulation accordingly. Also, the stimulation used in our study was restricted to a single site. However, memory processes are distributed across different brain regions and the most effective strategy would likely involve stimulation of multiple regions to produce more of a

211 network effect [29, 70] or an adaptive approach using closed-loop stimulation based on the state 212 of the network [71, 72]

213 In conclusion, we show that periodic pulse stimulation of cortex through subdural electrodes at low 214 frequency can enhance working memory. Despite the limitations, the study provides valuable 215 insights into the feasibility of using oscillations as brain stimulation targets. The importance is 216 highlighted by the emerging interest in using invasive recordings and electrical stimulation to 217 understand and alter pathological signatures of brain activity, whether it be neurological disorders,

218 like epilepsy and Parkinson's disease, or psychiatric disorders, like depression and obsessive219 compulsive disorder. Our results suggest that the same technology could be leveraged to also 220 address cognitive impairment.

\section{Experimental Procedures}

\section{ECoG Data Collection and Direct Cortical Stimulation}

224 All experimental procedures were approved by the Institutional Review Board of University of 225 North Carolina at Chapel Hill (IRB Number 13-2710) and written informed consent was obtained 
from the participant. The participants underwent implantation of intracranial EEG electrodes followed by long-term monitoring at the Epilepsy Monitoring Unit in UNC Neuroscience hospital for surgical resection planning.

Strips of electrodes were implanted over bilateral frontal, temporal and parietal lobes as shown in

Figure 1A. Depth electrodes were implanted in bilateral parahippocampal gyri in P1 and strip electrodes were implanted over bilateral occipital lobe in P2 (not shown in figure). The locations of the electrodes were completely dictated by the clinical needs of the participant. The electrodes, 4 $\mathrm{mm}$ in diameter (2.5 mm exposed), were made of platinum-iridium alloy and embedded in silicone

234 (Ad-Tech Medical, Racine, Wisconsin, United States). The electrodes in each strip were separated 235 by $10 \mathrm{~mm}$. Signals from electrodes that were over seizure foci (Table 1) were excluded from analysis.

237 ECoG data from participant P1 was recorded using a 128-channel acquisition system (Aura LTM

238 64, Grass Technologies, Warwick, Rhode Island, United States) at $800 \mathrm{~Hz}$ sampling rate.

239 Electrical stimulation consisted of 5 second train of biphasic pulses, $2 \mathrm{~mA}$ in amplitude, $400 \mu \mathrm{s}$ in 240 duration and $10 \mathrm{~Hz}$ in frequency. The pulses were generated by a cortical stimulator (S12x cortical 241 stimulator, Grass Technologies, Warwick, Rhode Island, United States) and applied between pairs 242 of adjacent electrodes (blue electrodes in Figure 1A).

243 ECoG data from participants P2 and P3 were recorded using a different 128-channel EEG system 244 (NetAmps 410, Electrical Geodesics Inc, Eugene, Oregon, United States) at $1000 \mathrm{~Hz}$ sampling 245 rate. Stimulation was delivered using Cerestim M96 cortical stimulator (Blackrock Microsystems, 246 Salt Lake City, Utah, United States). Stimulation parameters (except frequency) remained the 247 same as in P1 except for the duration which was adjusted to encompass the encoding and 248 retention epochs. 


\begin{tabular}{|l|l|l|l|l|l|l|}
\hline Participant & Age & Sex & Handedness & Clinical Seizure Focus & $\begin{array}{l}\text { Stimulation } \\
\text { Frequency }\end{array}$ & $\begin{array}{l}\text { Number of } \\
\text { Trials }\end{array}$ \\
\hline P1 & 23 & F & R & $\begin{array}{l}\text { Bilateral } \\
\text { parahippocampal gyri }\end{array}$ & $10 \mathrm{~Hz}$ & $\begin{array}{l}\text { 24 Sham, 13 } \\
\text { Stimulation }\end{array}$ \\
\hline P2 & 57 & M & R & $\begin{array}{l}\text { Bilateral inferior } \\
\text { occipital, posterior } \\
\text { temporal }\end{array}$ & $9 \mathrm{~Hz}$ & $\begin{array}{l}\text { 27 Sham, 26 } \\
\text { Stimulation }\end{array}$ \\
\hline P3 & 26 & M & R & $\begin{array}{l}\text { Unknown Seizure } \\
\text { Focus }\end{array}$ & $5 \mathrm{~Hz}$ & $\begin{array}{l}30 \text { Sham, 30 } \\
\text { Stimulation }\end{array}$ \\
\hline
\end{tabular}

We adopted a classical Sternberg working memory task previously used in other ECoG studies $[34,35,73]$ (Figure 1C). The task consisted of 3 epochs. In the first epoch, lists of 3 to 5 pseudorandomly chosen letters from the English alphabet were presented sequentially. This was termed the encoding epoch and each alphabet was displayed for 500 ms with 200 ms between each alphabet (the inter-alphabet interval was not present for P2 and P3). Following this, was a retention epoch where a blank screen was presented for 1 second. The final epoch was the retrieval epoch where a single probe (another English alphabet) was shown for 5 seconds and the participants had to indicate if they thought that the probe was present in the list by pressing a specified key on the keyboard. If they did not think the probe was present in the list, they did not have to press any key. The task was programmed in Matlab using Psychtoolbox [74] and presented in a laptop. For the experiment in which P1 participated, triggers from the cortical stimulator were detected by an ethernet DAQ (National instruments, Austin, TX, USA) connected to the task computer and used to initiate trials. Sham trials, in which no electrical pulses were delivered, were initiated using a pulse generator and were randomly interleaved with stimulation trials. For the experimental session in which P2 and P3 participated, triggers were generated within the Psychtoolbox task code and sent to Cerestim through the ethernet DAQ. In sham trials, 
271 no triggers were sent to Cerestim. Stimulation was applied for 5 seconds in P1 and the duration of

272 encoding and retention epochs in P2 and P3. In P1 electrodes over right SFG and bilateral

273 temporal cortices were stimulated as well. However, the low number of stimulation trials did not

274 allow any meaningful analysis to be performed and hence was not included in the study here. In

275 P2, a pair of electrode over right SFG was stimulated and the results are not included here.

276 Participants P2 and P3 completed 2 sessions - a baseline session and a stimulation session. The

277 baseline session did not include any stimulation and consisted of 40 trials of two different list

278 lengths to assess the baseline performance level as well as determine the parameters for the

279 stimulation session.

280 Data Analysis

281 Data analysis was performed using custom written Matlab scripts (The MathWorks Inc., Natick, 282 MA, United States). The recording setup consisted of switching circuits designed to protect the 283 amplifier during stimulation which prevented recording of data from stimulating electrodes. Hence, 284 data from stimulating electrodes were not included in the analysis.

285 Stimulation artifacts, present in channels adjacent to stimulated electrodes, were removed using

286 an independent component analysis (ICA) based approach (Figure S). Since artifacts were

287 observed as stereotypical waveforms, ICA resulted in components that contained only artifact 288 waveforms which were then rejected, and the remaining components were used to reconstruct 289 artifact free signals. We used the infomax algorithm [75] available as a part of EEGLab toolbox 290 [76] for computing independent components. Following artifact suppression, the signals were low 291 pass filtered with an FIR filter (cutoff frequency $50 \mathrm{~Hz}$ ) and re-referenced to common average.

292 Signal power spectra was computed with a multi-taper fft based approach using Chronux toolbox 293 [77]. To quantify the change induced by stimulation, modulation index was computed as

$$
\text { Modulation Index }=\frac{\left(\overline{\boldsymbol{S}}_{e}-\overline{\boldsymbol{S}}_{\mathrm{b}}\right)}{\left(\overline{\boldsymbol{S}}_{e}+\overline{\boldsymbol{S}}_{\mathrm{b}}\right)}
$$


295 Where $\overline{\boldsymbol{S}}_{\boldsymbol{e}}$ and $\overline{\boldsymbol{S}}_{\boldsymbol{b}}$ are average power in specified frequency band in specific epoch (task, encoding or retention) and baseline epoch respectively. The baseline epoch was defined as 5 second interval before the beginning of encoding epoch.

298 Time-frequency representations were computed by convolving Morlet wavelets with the time series of each trial. Event related spectral perturbation was calculated as

$$
E R S P=\log _{10}\left(\frac{S_{e}}{\bar{S}_{b}}\right)
$$

301 Where $\boldsymbol{S}_{\boldsymbol{e}}$ is the spectra at each time point within an epoch and $\overline{\boldsymbol{S}}_{\boldsymbol{b}}$ is the average power in the 302 baseline epoch.

\section{Statistics}

304 All statistical analyses were performed using R. Linear mixed effects models were fitted using the 305 Imertest package [78] which uses Satterthwaite's approximation to degrees of freedom to 306 determine the F statistics of the fixed effects.

307 For the effect of list length on reaction times, we fitted a linear model with reaction time as 308 dependent variable and list length as the factor for each participant separately. For the effect of list length on modulation indices, we fitted linear mixed model with modulation index as the dependent variable and list length as the fixed factor and participant and electrodes as nested random

311 factors. To study the effect of stimulation on reaction time, we fitted a linear mixed model with

312 reaction time as dependent variable and stimulation as fixed factors and participant as the random 313 factor. As post hoc analysis we performed a two-sample t-test to compare the difference between 314 reaction times during sham and stimulation trials for each participant. To study the effect of 315 stimulation on modulation index, we fitted linear mixed models with modulation index as 316 dependent variable, stimulation as fixed factor and electrodes and participants as nested random

317 factors and also with modulation index as dependent variable and list length (3 levels) and 318 stimulation regions (3 levels - sham, frontal region, temporal region) as fixed factors and 319 electrodes as a random factor. As post-hoc analysis, we performed paired t-tests. 
3D Slicer [79] was used to analyze and extract electrode locations from CT images obtained after implantation of subdural electrodes. The post-operative MRI was co-registered to post-operative CT in Slicer followed by registering to standard MNI atlas [80]. Skull stripping was performed using ROBEX [81], and the gray matter and white matter were then segmented using ITK-Snap [82]. The surface model of the MNI atlas brain was generated using Slicer and used for visualization purposes. The anatomical locations of the electrodes were determined by co-registering the MRI Image to the MNI Atlas [83], recomputing electrode locations in the MNI space, transforming these locations to Talairach space, and using the Talairach Client [84] to obtain the label of the gray matter nearest to the coordinate representing electrode location.

\section{Acknowledgments}

332 The authors thank the members of the Frohlich Lab for their valuable input, with special thanks to 333 Sangtae Ahn for verifying the accuracy of the codes used for analysis and providing valuable 334 feedback on the manuscript. The authors also thank the EEG technicians at the UNC Epilepsy monitoring unit for their generous help with the ECoG recordings.

\section{Funding}

338 Research reported in this publication was supported in part by the National Institute of Mental Health 339 of the National Institutes of Health under Award Numbers R01MH101547 and R21MH105557, 340 National Institute of Neurological Disorders and Stroke of the National Institutes of Health under 341 award number R21NS094988-01A1, Translational Team Science Award (TTSA) with funding 342 provided by the UNC School of Medicine and the National Center for Advancing Translational 343 Sciences (NCATS), National Institutes of Health, through Grant Award Number UL1TR001111, 344 Helen Lyng White Postdoctoral Fellowship (S.A.) and Swiss National Science Foundation (grant 345 P300PA_164693; C.L.). The content is solely the responsibility of the authors and does not necessarily represent the official views of the National Institutes of Health. 


\section{Supplementary Figures}

A

\section{Before Artifact Suppression}

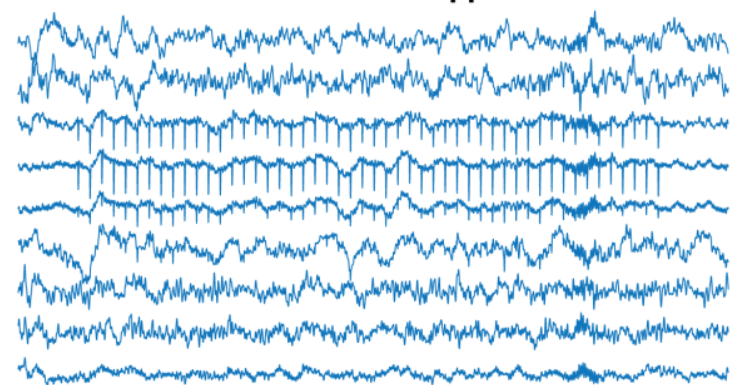

\section{C}

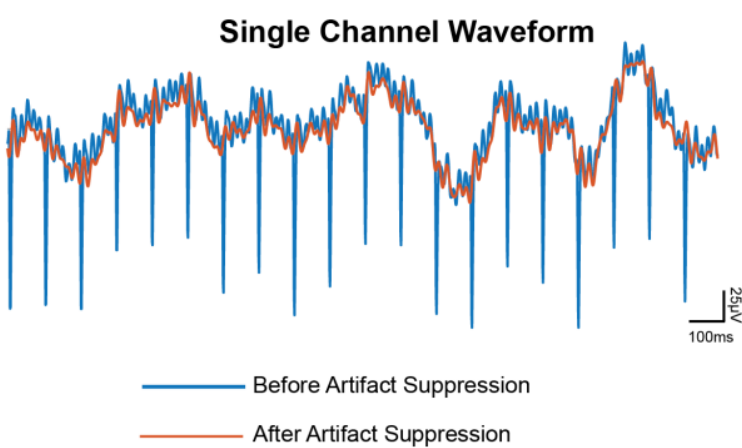

B
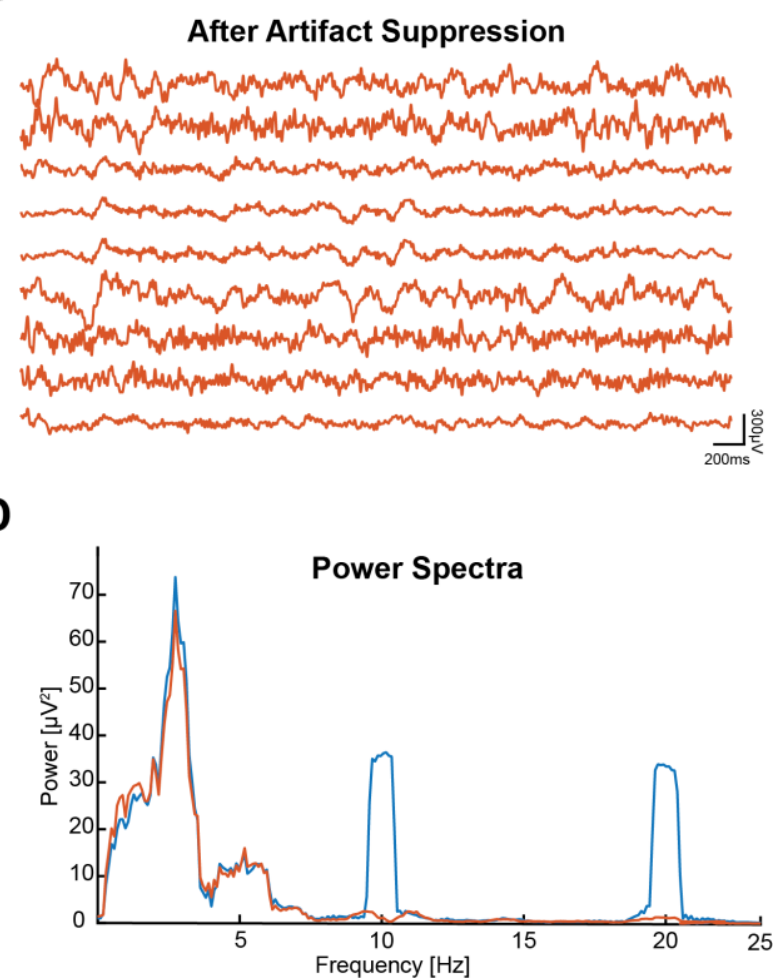

Figure S1: An example illustrating performance of ICA based artifact suppression algorithm. (A) Raw signal from channels adjacent to stimulation channels before artifact suppression (B) Signal after removal of stimulation artifacts. Visually, the artifact has been reduced to noise level. (C) Trace from a single channel highlighting the suppression of artifact waveform (D) Power spectra computed from the signal shown in $(\mathrm{C})$ before and artifact suppression. 
bioRxiv preprint doi: https://doi.org/10.1101/302588; this version posted April 17, 2018. The copyright holder for this preprint (which was not certified by peer review) is the author/funder, who has granted bioRxiv a license to display the preprint in perpetuity. It is made available under aCC-BY-NC-ND 4.0 International license.

\section{Bibliography}

352

353

354

355

356

357

358

359

360

361

362

363

364

365

366

367

368

369

370

371

372

373

374

375

376

377

378

379

380

381

382

383

384

385

386

387

388

389

390

391

392

393

394

395

396

397

398

399

400

401

1. Unsworth, N., et al., Working memory and fluid intelligence: capacity, attention control, and secondary memory retrieval. Cogn Psychol, 2014. 71: p. 1-26.

2. Ackerman, P.L., M.E. Beier, and M.O. Boyle, Working memory and intelligence: the same or different constructs? Psychol Bull, 2005. 131(1): p. 30-60.

3. Forbes, N.F., et al., Working memory in schizophrenia: a meta-analysis. Psychol Med, 2009. 39(6): p. 889-905.

4. Campo, P., et al., Network reconfiguration and working memory impairment in mesial temporal lobe epilepsy. Neuroimage, 2013. 72: p. 48-54.

5. Lee, J. and S. Park, Working memory impairments in schizophrenia: a metaanalysis. J Abnorm Psychol, 2005. 114(4): p. 599-611.

6. Snyder, H.R., Major Depressive Disorder Is Associated With Broad Impairments on Neuropsychological Measures of Executive Function: A Meta-Analysis and Review. Psychological Bulletin, 2013. 139(1): p. 81-132.

7. Uhlhaas, P.J. and W. Singer, Neuronal dynamics and neuropsychiatric disorders: toward a translational paradigm for dysfunctional large-scale networks. Neuron, 2012. 75(6): p. 963-80.

8. Braver, T.S., et al., A parametric study of prefrontal cortex involvement in human working memory. Neuroimage, 1997. 5(1): p. 49-62.

9. Awh, E., E.E. Smith, and J. Jonides, Human rehearsal processes and the frontal lobes: PET evidence. Structure and Functions of the Human Prefrontal Cortex, 1995. 769: p. 97-117.

10. Cornette, L., et al., The neural substrate of orientation working memory. Journal of Cognitive Neuroscience, 2001. 13(6): p. 813-828.

11. Wager, T.D. and E.E. Smith, Neuroimaging studies of working memory: a metaanalysis. Cogn Affect Behav Neurosci, 2003. 3(4): p. 255-74.

12. D'Esposito, M., From cognitive to neural models of working memory. Philos Trans R Soc Lond B Biol Sci, 2007. 362(1481): p. 761-72.

13. Ranganath, C., et al., Inferior temporal, prefrontal, and hippocampal contributions to visual working memory maintenance and associative memory retrieval. J Neurosci, 2004. 24(16): p. 3917-25.

14. Curtis, C.E., Prefrontal and parietal contributions to spatial working memory. Neuroscience, 2006. 139(1): p. 173-80.

15. Curtis, C.E. and M. D'Esposito, Persistent activity in the prefrontal cortex during working memory. Trends in Cognitive Sciences, 2003. 7(9): p. 415-423.

16. du Boisgueheneuc, F., et al., Functions of the left superior frontal gyrus in humans: a lesion study. Brain, 2006. 129(Pt 12): p. 3315-28.

17. Krause, C.M., et al., The effects of memory load on event-related EEG desynchronization and synchronization. Clin Neurophysiol, 2000. 111(11): p. 20718.

18. Hsieh, L.T. and C. Ranganath, Frontal midline theta oscillations during working memory maintenance and episodic encoding and retrieval. Neuroimage, 2014. 85 Pt 2: p. 721-9.

19. Jensen, O., et al., Oscillations in the alpha band (9-12 Hz) increase with memory load during retention in a short-term memory task. Cereb Cortex, 2002. 12(8): $\mathrm{p}$. 877-82.

20. Tesche, C.D. and J. Karhu, Theta oscillations index human hippocampal activation during a working memory task. Proc Natl Acad Sci U S A, 2000. 97(2): p. 919-24.

21. Gevins, A., et al., High-resolution EEG mapping of cortical activation related to working memory: effects of task difficulty, type of processing, and practice. Cereb Cortex, 1997. 7(4): p. 374-85. 
bioRxiv preprint doi: https://doi.org/10.1101/302588; this version posted April 17, 2018. The copyright holder for this preprint (which was not certified by peer review) is the author/funder, who has granted bioRxiv a license to display the preprint in perpetuity. It is made available under aCC-BY-NC-ND 4.0 International license.

402 22. Mottaghy, F.M., et al., Segregation of areas related to visual working memory in the prefrontal cortex revealed by rTMS. Cerebral Cortex, 2002. 12(4): p. 369-375.

23. Oliveri, M., et al., Parieto-frontal interactions in visual-object and visual-spatial working memory: evidence from transcranial magnetic stimulation. Cereb Cortex, 2001. 11(7): p. 606-18.

24. Polania, R., et al., The importance of timing in segregated theta phase-coupling for cognitive performance. Curr Biol, 2012. 22(14): p. 1314-8.

25. Jausovec, N., K. Jausovec, and A. Pahor, The influence of theta transcranial alternating current stimulation (tACS) on working memory storage and processing functions. Acta Psychol (Amst), 2014. 146: p. 1-6.

26. Vosskuhl, J., R.J. Huster, and C.S. Herrmann, Increase in short-term memory capacity induced by down-regulating individual theta frequency via transcranial alternating current stimulation. Front Hum Neurosci, 2015. 9: p. 257.

27. Violante, I.R., et al., Externally induced frontoparietal synchronization modulates network dynamics and enhances working memory performance. Elife, 2017. 6.

28. Borchers, S., et al., Direct electrical stimulation of human cortex - the gold standard for mapping brain functions? Nat Rev Neurosci, 2012. 13(1): p. 63-70.

29. Kim, K., A.D. Ekstrom, and N. Tandon, A network approach for modulating memory processes via direct and indirect brain stimulation: Toward a causal approach for the neural basis of memory. Neurobiol Learn Mem, 2016.

30. Suthana, N. and I. Fried, Deep brain stimulation for enhancement of learning and memory. Neuroimage, 2014. 85 Pt 3: p. 996-1002.

31. Suthana, N., et al., Memory enhancement and deep-brain stimulation of the entorhinal area. N Engl J Med, 2012. 366(6): p. 502-10.

32. Kucewicz, M.T., et al., Evidence for verbal memory enhancement with electrical brain stimulation in the lateral temporal cortex. Brain, 2018.

33. Alagapan, S., et al., Modulation of Cortical Oscillations by Low-Frequency Direct Cortical Stimulation Is State-Dependent. PLoS Biol, 2016. 14(3): p. e1002424.

34. Raghavachari, S., et al., Gating of human theta oscillations by a working memory task. J Neurosci, 2001. 21(9): p. 3175-83.

35. Meltzer, J.A., et al., Effects of working memory load on oscillatory power in human intracranial EEG. Cereb Cortex, 2008. 18(8): p. 1843-55.

36. Jensen, O. and C.D. Tesche, Frontal theta activity in humans increases with memory load in a working memory task. Eur J Neurosci, 2002. 15(8): p. 1395-9.

37. Owen, A.M., et al., N-back working memory paradigm: a meta-analysis of normative functional neuroimaging studies. Hum Brain Mapp, 2005. 25(1): p. 4659.

38. D'Esposito, M. and B.R. Postle, The cognitive neuroscience of working memory. Annu Rev Psychol, 2015. 66: p. 115-42.

39. Rypma, B., et al., Load-dependent roles of frontal brain regions in the maintenance of working memory. Neuroimage, 1999. 9(2): p. 216-26.

40. Harms, M.P., et al., Structure-function relationship of working memory activity with hippocampal and prefrontal cortex volumes. Brain Struct Funct, 2013. 218(1): p. 173-86.

41. Li, W., et al., Subregions of the human superior frontal gyrus and their connections. Neuroimage, 2013. 78: p. 46-58.

42. Cole, M.W. and W. Schneider, The cognitive control network: Integrated cortical regions with dissociable functions. Neuroimage, 2007. 37(1): p. 343-60.

43. Harding, I.H., et al., Effective connectivity within the frontoparietal control network differentiates cognitive control and working memory. Neuroimage, 2015. 106: p. 144-53. 
bioRxiv preprint doi: https://doi.org/10.1101/302588; this version posted April 17, 2018. The copyright holder for this preprint (which was not certified by peer review) is the author/funder, who has granted bioRxiv a license to display the preprint in perpetuity. It is made available under aCC-BY-NC-ND 4.0 International license.

453

454

455

456

457

458

459

460

461

462

463

464

465

466

467

468

469

470

471

472

473

474

475

476

477

478

479

480

481

482

483

484

485

486

487

488

489

490

491

492

493

494

495

496

497

498

499

500

501

502

503

44. Engle, R.W. and M.J. Kane, Executive attention, working memory capacity, and a two-factor theory of cognitive control. Psychology of Learning and Motivation:

Advances in Research and Theory, Vol 44, 2004. 44: p. 145-199.

45. Jensen, O. and J.E. Lisman, An oscillatory short-term memory buffer model can account for data on the Sternberg task. J Neurosci, 1998. 18(24): p. 10688-99.

46. Sauseng, P., et al., Brain oscillatory substrates of visual short-term memory capacity. Curr Biol, 2009. 19(21): p. 1846-52.

47. Mitchell, D.J., et al., Frontal-midline theta from the perspective of hippocampal "theta". Prog Neurobiol, 2008. 86(3): p. 156-85.

48. Fell, J., et al., Memory modulation by weak synchronous deep brain stimulation: a pilot study. Brain Stimul, 2013. 6(3): p. 270-3.

49. Lacruz, M.E., et al., Single pulse electrical stimulation of the hippocampus is sufficient to impair human episodic memory. Neuroscience, 2010. 170(2): p. 62332.

50. Coleshill, S.G., et al., Material-specific recognition memory deficits elicited by unilateral hippocampal electrical stimulation. J Neurosci, 2004. 24(7): p. 1612-6.

51. Jacobs, J., et al., Direct Electrical Stimulation of the Human Entorhinal Region and Hippocampus Impairs Memory. Neuron, 2016. 92(5): p. 983-990.

52. Koubeissi, M.Z., et al., Low-frequency electrical stimulation of a fiber tract in temporal lobe epilepsy. Ann Neurol, 2013. 74(2): p. 223-31.

53. Miller, J.P., et al., Visual-spatial memory may be enhanced with theta burst deep brain stimulation of the fornix: a preliminary investigation with four cases. Brain, 2015. 138(Pt 7): p. 1833-42.

54. Luber, B., et al., Facilitation of performance in a working memory task with rTMS stimulation of the precuneus: Frequency-and time-dependent effects. Brain Research, 2007. 1128(1): p. 120-129.

55. Guse, B., et al., The effect of long-term high frequency repetitive transcranial magnetic stimulation on working memory in schizophrenia and healthy controls--a randomized placebo-controlled, double-blind fMRI study. Behav Brain Res, 2013. 237: p. 300-7.

56. Bagherzadeh, Y., et al., Repetitive transcranial magnetic stimulation of the dorsolateral prefrontal cortex enhances working memory. Exp Brain Res, 2016. 234(7): p. 1807-18.

57. Blumenfeld, R.S., T.G. Lee, and M. D'Esposito, The effects of lateral prefrontal transcranial magnetic stimulation on item memory encoding. Neuropsychologia, 2014. 53: p. 197-202.

58. Esslinger, C., et al., Induction and Quantification of Prefrontal Cortical Network Plasticity Using $5 \mathrm{~Hz}$ rTMS and fMRI. Human Brain Mapping, 2014. 35(1): p. 140151.

59. Yamanaka, K., et al., Effect of parietal transcranial magnetic stimulation on spatial working memory in healthy elderly persons--comparison of near infrared spectroscopy for young and elderly. PLoS One, 2014. 9(7): p. e102306.

60. Yamanaka, K., et al., Transcranial magnetic stimulation of the parietal cortex facilitates spatial working memory: near-infrared spectroscopy study. Cereb Cortex, 2010. 20(5): p. 1037-45.

61. Hoy, K.E., et al., Enhancement of Working Memory and Task-Related Oscillatory Activity Following Intermittent Theta Burst Stimulation in Healthy Controls. Cereb Cortex, 2016. 26(12): p. 4563-4573.

62. Postle, B.R., et al., Repetitive transcranial magnetic stimulation dissociates working memory manipulation from retention functions in the prefrontal, but not posterior parietal, cortex. J Cogn Neurosci, 2006. 18(10): p. 1712-22. 
bioRxiv preprint doi: https://doi.org/10.1101/302588; this version posted April 17, 2018. The copyright holder for this preprint (which was not certified by peer review) is the author/funder, who has granted bioRxiv a license to display the preprint in perpetuity. It is made available under aCC-BY-NC-ND 4.0 International license.

504

505

506

507

508

509

510

511

512

513

514

515

516

517

518

519

520

521

522

523

524

525

526

527

528

529

530

531

532

533

534

535

536

537

538

539

540

541

542

543

544

545

546

547

548

549

550

551

552

553

554

555

63. Osaka, N., et al., Transcranial magnetic stimulation (TMS) applied to left dorsolateral prefrontal cortex disrupts verbal working memory performance in humans. Neurosci Lett, 2007. 418(3): p. 232-5.

64. Gagnon, G., et al., Paired-pulse transcranial magnetic stimulation over the dorsolateral prefrontal cortex interferes with episodic encoding and retrieval for both verbal and non-verbal materials. Brain Res, 2010. 1344: p. 148-58.

65. Mottaghy, F.M., Interfering with working memory in humans. Neuroscience, 2006. 139(1): p. 85-90.

66. Albouy, P., et al., Selective Entrainment of Theta Oscillations in the Dorsal Stream Causally Enhances Auditory Working Memory Performance. Neuron, 2017.

67. Thut, G., P.G. Schyns, and J. Gross, Entrainment of perceptually relevant brain oscillations by non-invasive rhythmic stimulation of the human brain. Front Psychol, 2011. 2: p. 170.

68. Helfrich, R.F., et al., Entrainment of brain oscillations by transcranial alternating current stimulation. Curr Biol, 2014. 24(3): p. 333-9.

69. Li, G., C.S. Henriquez, and F. Frohlich, Unified thalamic model generates multiple distinct oscillations with state-dependent entrainment by stimulation. PLoS Comput Biol, 2017. 13(10): p. e1005797.

70. Kim, K., et al., Network-based brain stimulation selectively impairs spatial retrieval. Brain Stimul, 2018. 11(1): p. 213-221.

71. Ezzyat, Y., et al., Direct Brain Stimulation Modulates Encoding States and Memory Performance in Humans. Curr Biol, 2017. 27(9): p. 1251-1258.

72. Ezzyat, Y., et al., Closed-loop stimulation of temporal cortex rescues functional networks and improves memory. Nat Commun, 2018. 9(1): p. 365.

73. Raghavachari, S., et al., Theta oscillations in human cortex during a workingmemory task: evidence for local generators. J Neurophysiol, 2006. 95(3): p. 16308.

74. Brainard, D.H., The Psychophysics Toolbox. Spat Vis, 1997. 10(4): p. 433-6.

75. Lee, T.W., et al., A unifying information-theoretic framework for independent component analysis. Computers \& Mathematics with Applications, 2000. 39(11): $p$. $1-21$.

76. Delorme, A. and S. Makeig, EEGLAB: an open source toolbox for analysis of single-trial EEG dynamics including independent component analysis. J Neurosci Methods, 2004. 134(1): p. 9-21.

77. Bokil, H., et al., Chronux: a platform for analyzing neural signals. J Neurosci Methods, 2010. 192(1): p. 146-51.

78. Kuznetsova, A., P.B. Brockhoff, and R.H.B. Christensen, ImerTest Package: Tests in Linear Mixed Effects Models. Journal of Statistical Software, 2017. 82(13): p. 126.

79. Fedorov, A., et al., 3D Slicer as an image computing platform for the Quantitative Imaging Network. Magn Reson Imaging, 2012. 30(9): p. 1323-41.

80. Fonov, V.S., et al., Unbiased nonlinear average age-appropriate brain templates from birth to adulthood. Neurolmage, 2009. 47, Supplement 1: p. S102.

81. Iglesias, J.E., et al., Robust brain extraction across datasets and comparison with publicly available methods. IEEE Trans Med Imaging, 2011. 30(9): p. 1617-34.

82. Yushkevich, P.A., et al., User-guided 3D active contour segmentation of anatomical structures: significantly improved efficiency and reliability. Neuroimage, 2006. 31(3): p. 1116-28.

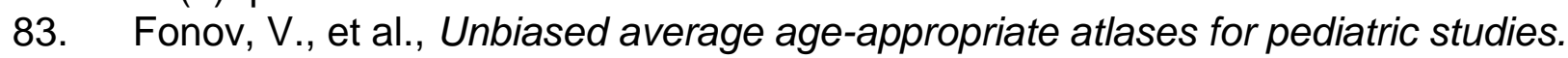
Neuroimage, 2011. 54(1): p. 313-27.

84. Lancaster, J.L., et al., Automated Talairach atlas labels for functional brain mapping. Hum Brain Mapp, 2000. 10(3): p. 120-31. 